\title{
Associations of Food Addiction and Non-suicidal Self-injury amongst Women with an Eating Disorder: A Common Strategy for Regulating Emotions?
}

Lily Carlson ${ }^{1 \#}$, Trevor Steward ${ }^{2,4 \#}$, Zaida Agüera, ${ }^{2,4}$, Gemma Mestre-Bach, ${ }^{2,4}$, Pablo Magaña $^{2,5}$, Roser Granero ${ }^{4,6}$, Susana Jiménez-Murcia ${ }^{2,4,7}$, Laurence Claes ${ }^{8,9}$, Ashley N. Gearhardt $^{3}$, José M. Menchón ${ }^{2,7,10}$, Fernando Fernández-Aranda ${ }^{2,4,7 *}$

${ }^{1}$ School of Public Health, University of Michigan, Ann Arbor, MI, USA.

${ }^{2}$ Department of Psychiatry, Bellvitge University Hospital -IDIBELL, Barcelona, Spain.

${ }^{3}$ Department of Psychology, University of Michigan, Ann Arbor, MI, USA.

${ }^{4}$ Ciber Fisiopatologia Obesidad y Nutrición (CIBEROBN), Instituto Salud Carlos III, Barcelona, Spain

${ }^{5}$ Department of Law, Pompeu Fabra University. Barcelona, Spain.

${ }^{6}$ Departament de Psicobiologia $i$ Metodologia de les Ciències de la Salut. Universitat Autònoma de Barcelona, Barcelona, Spain.

${ }^{7}$ Department of Clinical Sciences, School of Medicine, University of Barcelona, Spain

${ }^{8}$ Faculty of Psychology and Educational Sciences, University of Leuven, Leuven, Belgium

${ }^{9}$ Faculty of Medicine and Health Sciences, University of Antwerp, Antwerp, Belgium

${ }^{10}$ Ciber Salud Mental (CIBERSAM), Instituto Salud Carlos III, Barcelona, Spain.

\section{*Correspondence:}

Fernando Fernández-Aranda, Eating Disorders Unit-Department of Psychiatry and CIBERObn, University Hospital of Bellvitge-IDIBELL, c/ Feixa Llarga s/n, 08907_ Barcelona, Spain. Tel. +34-93-2607227. E-mail: ffernandez@bellvitgehospital.cat This is the author manuscript accepted for publication and has undergone full peer review but has not been through the copyediting, typesetting, pagination and proofreading process, which \#Shared first authorship to differences between this version and the Version of Record. Please cite this article as doi: 10.1002/erv.2646

This article is protected by copyright. All rights reserved. 
Running title: Food addiction and NSSI

\section{Funding:}

This manuscript and research was supported by grants from the Ministerio de Economía y Competitividad (PSI2015-68701-R), Ministerio de Sanidad, Servicios Sociales e Igualdad (PR338/17), Instituto de Salud Carlos III (ISCIII) (FIS PI14/00290 and PI17/01167) and cofunded by FEDER funds /European Regional Development Fund (ERDF), a way to build Europe. CIBERObn and CIBERSAM are both initiatives of ISCIII. The funders had no role in the study design, data collection and analysis, decision to publish, or preparation of the manuscript. This research was supported by a PNSD (PR338/17-MSSSI) grant. GMB is supported by a predoctoral AGAUR grant (2018 FI_B2 00174), co-financed by the European Social Fund, with the support of the Secretaria d'Universitats i Recerca del Departament d'Economia i Coneixement de la Generalitat de Catalunya.

Conflict of interest: The authors declare no conflict of interest. 


\begin{abstract}
Objective: We examined the association between lifetime non-suicidal self-injury (NSSI), emotion regulation, and food addiction $(F A)$ in women $(n=220)$ with eating disorders (ED) compared to $(\mathrm{n}=121)$ healthy controls $(\mathrm{HC})$. Method: Participants were assessed via face-toface interviews for ED diagnosis and lifetime NSSI. FA was assessed with Yale Food Addiction Scale (YFAS 2.0) and emotion regulation using the Difficulty in Emotion Regulation Scale (DERS). Results: The prevalence of FA was significantly higher among women with an ED when compared with $\mathrm{HC}(75.9 \%$ vs. $4.1 \%, \mathrm{p}<.001)$. Similarly, subjects presenting FA, showed a high prevalence of lifetime NSSI, in both ED and HC $(40.7 \%$ and $60.0 \%$, respectively). Our predictive model revealed FA and DERS total scores as indicators of the presence of lifetime NSSI independent of group assignment, ED diagnosis, and age. Conclusions: These findings suggest a shared etiology between ED, NSSI, and FA, explained possibly in part by emotion-regulation deficits.
\end{abstract}




\section{Highlights}

- The associations between non-suicidal self-injury (NSSI) and food addiction (FA) in women with eating disorders and healthy controls were positive.

- Subjects who presented FA, showed also higher prevalence of lifetime NSSI, in both ED as in $\mathrm{HC}$ (40.6\% vs. $60.0 \%$ respectively).

- FA and DERS Total scores were significant predictors of life-time NSSI.

Keywords: food addiction, non-suicidal self-injury, eating disorders, self-harm behaviors, risk factors. 


\section{INTRODUCTION}

Within the past decade, there has been a growing body of research investigating the existence of food addiction (FA) as a psychological disorder. FA posits that individuals experience addictive-like behaviors and cognitions, comparable to other substance use disorders, in response to hyperpalatable, highly processed foods (Gearhardt, Davis, Kuschner, \& Brownell, 2011; Meule \& Gearhardt, 2014).

The Yale Food Addiction Scale 2.0 (YFAS), a self-report questionnaire based upon the Diagnostic and Statistical Manual of Mental Disorders (APA, 2013) criteria for substance use disorders is the most widely used tool to assess FA (Schulte \& Gearhardt, 2017).

The prevalence of FA in the general population ranges from $11 \%$ to $40 \%$, with middle-age, female, and low socioeconomic populations experiencing the highest incidence (Granero et al., 2018; Pursey, Stanwell, Gearhardt, Collins, \& Burrows, 2014). The prevalence of FA is higher among individuals with an eating disorder (ED) compared to healthy controls (HC). In a sample of Spanish women with an ED, the prevalence of FA was $72.8 \%$ versus $2.4 \%$ amongst $\mathrm{HC}$ (Granero et al., 2014). Furthermore, FA is linked to binge eating behaviors as evidenced by the elevated prevalence of FA amongst individuals with binge eating disorder (BED) and bulimia nervosa (BN) (Gearhardt et al., 2012a; Meule, von Rezori, \& Blechert, 2014). In line with the aforementioned results, Granero et al. (2014) found that individuals with $\mathrm{BN}$ and BED exhibited increased endorsement of FA symptoms as compared to individuals with anorexia nervosa (AN) and eating disorders not otherwise specified (EDNOS) (Granero et al., 2014).

FA has also been associated with other forms of psychopathology, such as a major depressive episode, a bipolar disorder and skin picking disorder (Nunes-Neto et al., 2018). Furthermore, FA has been associated with higher negative affect, lower self-esteem, and 
higher emotion dysregulation amongst individuals with an ED (Gearhardt et al., 2012; Pisetsky, Haynos, Lavender, Crow, \& Peterson, 2017; Wolz, Granero, \& Fernández-Aranda, 2017). Likewise, affective instability has been linked to dysregulated eating behaviors (i.e. bingeing and purging episodes) in women with BN (Berner et al., 2017; Wolz et al., 2016). FA has also been associated with impairments in executive functioning, namely impulsivity: individuals with FA in a sample of individuals with an ED diagnosis scored higher on measures of negative urgency and lack of perseverance, indicating impulsive characteristics within this ED population with FA (Granero et al., 2018; Pivarunas \& Conner, 2015; Wolz et al., 2016).

Non-suicidal self-injury (NSSI) is defined as the direct and intentional destruction of one's own body tissue without suicidal intent, such as cutting, burning, biting, and scratching the skin (Zetterqvist, 2015). Despite the distinction between NSSI and suicidal behaviors, the presence of NSSI is a risk factor for suicide (Claes \& Muehlenkamp, 2014). Furthermore, the presence of NSSI has been associated with other mental health disorders, such as depression, anxiety, PTSD, and features of some personality disorders (Claes \& Muehlenkamp, 2014). NSSI is often interpreted as emotion regulation behavior and individuals who engage in NSSI often report higher levels of emotional dysregulation than individuals without NSSI (Andover \& Morris, 2014; Kranzler, Fehling, Anestis, \& Selby, 2016; Vieira et al., 2018). Furthermore, the prevalence of NSSI amongst individuals with ED has been estimated between $14 \%$ to 68\% (Solano, Fernández-Aranda, Aitken, López, \& Vallejo, 2005; Svirko \& Hawton, 2007), with NSSI being associated with higher ED severity, general psychopathology and dysfunctional personality traits.

Given the significant overlap of individuals with FA and binge eating behaviors, it is reasonable to assume that individuals with FA may also be at higher risk for NSSI, as shown by the aforementioned data on NSSI among individuals with binge-type ED and the 
commonly reported difficulties in emotion regulation in both conditions (Gearhardt et al., 2012; Vieira et al., 2017). There is also evidence for the presence of impulsivity in individuals with FA and NSSI, further suggesting a potential association between these behaviors (Wolz, Granero, \& Fernández-Aranda, 2017). Furthermore, emotion dysregulation is also likely to serve as a mediator in the relationship between NSSI and FA. Emotional dysregulation has been found to be elevated in individuals with NSSI and it has been suggested that NSSI be understood as an inappropriate response to negative affect amongst individuals with disordered eating (Muehlenkamp, Peat, Claes, \& Smits, 2012; Yurkowski et al., 2017).

A better understanding of the relationship between FA, NSSI, and emotion regulation is essential for a more comprehensive understanding of risk factors for both FA and NSSI. Previously established associations between NSSI and binge-type ED, increased exhibition of dysregulated behavior amongst individuals with NSSI and ED, and an overlap between FA and binge-type disorders are indicative of several overlaps across behaviors (Gearhardt et al., 2012b; Islam et al., 2015; Steward et al., 2018; Vieira et al., 2017; Wolz, Granero, \& Fernández-Aranda, 2017). Furthermore, emotion dysregulation can be considered as a common trait among individuals with NSSI and FA (Muehlenkamp et al., 2012; Wolz et al., 2016; Yurkowski et al., 2017). Establishing a link between FA and NSSI and its underlying mechanisms could be advantageous in identifying individuals who may be at higher risk for either NSSI or FA, and insight in underlying mechanisms of this association allows for preventive interventions.

To the best of our knowledge, no study to date has examined the relationship between FA, emotion regulation and NSSI. In this study, we aim to compare the presence of NSSI in individuals with $\mathrm{ED}$ and $\mathrm{HC}$ with and without FA. Further, we will investigate the prevalence of NSSI among different ED subtypes. We will use a predictive model to determine which 
features best predict the occurrence of NSSI. Based on previous studies that indicate a higher prevalence of NSSI in binge eating/purging ED patients, we predicted a positive association between NSSI and FA. We also hypothesized that higher levels of emotion regulation difficulties would be positively linked to NSSI.

\section{Methods}

\subsection{Participants and Procedure}

The sample consisted of 220 adult female ED patients who were consecutive referrals to the Eating Disorders Unit at a University Bellvitge Hospital. HCs were recruited from the surrounding hospital community and included students from the university hospital setting who voluntarily chose to participate in the study as well as hospital employees and volunteers recruited by word of mouth. Only female HCs were included in our study sample. Diagnoses for EDs were originally made using the Diagnostic and Statistical Manual for Mental Disorders (DSM-IV-TR; APA, 2000) by means of a semi-structured interview (SCID-I; First, Spitzer, Gibbon, \& Williams, 2002) conducted by trained psychologists and psychiatrists. Diagnoses were re-codified post-hoc using DSM-5 criteria (APA, 2013). Males were excluded from the study as the number of men with EDs in our sample was too small for meaningful comparisons.

The Ethics Committee of our Institution (the Ethics Committee of Clinical Research of the participating University Hospital) approved the study, and all research was conducted in accordance with the latest version of the Helsinki Declaration. Signed informed consent was obtained from all participants.

\subsection{Assessment}

The presence/absence of NSSI was investigated by a 1-item question asking "Have you ever engaged in self-injury without the intent to die (such as self-cutting, burning, hitting, 
scratching, and hair pulling)?" (Islam et al., 2015). Using a single-item measure is common in NSSI research and has been shown to render consistent estimates of prevalence (Claes, Soenens, Vansteenkiste, \& Vandereycken, 2012; Gómez-Expósito et al., 2016; Muehlenkamp et al., 2012).

\subsubsection{Yale Food Addiction Scale 2.0 (YFAS 2.0) (Gearhardt, Corbin, \& Brownell, 2016)}

This self-report questionnaire consists of 35 items scored on an eight-level Likert scale (from $0=$ never to $7=$ every day) and is adapted to assess addictive eating behaviors based on DSM-5 substance-related and addictive disorders criteria (APA, 2013).

The Spanish YFAS 2.0 has been validated for a Spanish-speaking population and the scoring guidelines used in the original validation of the YFAS 2.0 were followed for this study (Granero et al., 2018). These scoring guidelines produces two scores: (a) a continuous symptom count score that reflects the number of fulfilled diagnostic criteria; and (b) a FA threshold based on the number of symptoms and self-reported clinically significant impairment or distress. This final score allows for the binary classification of FA (present versus absent). The Spanish version of the YFAS 2.0 has excellent accuracy of discriminating between $\mathrm{HC}$ and ED subsamples $(\kappa=0.75)$ and its internal consistency coefficient is excellent $(\alpha=0.94)$ (Granero et al., 2018).

\subsubsection{Eating Disorders Inventory-2 (EDI-2) (Garner, 1991)}

This is a reliable and valid 91-item self-report questionnaire that assesses different cognitive and behavioral ED characteristics. All of these items are answered on a six-point Likert scale and provide standardized subscale scores. In the current study, the total score was used as a measure of ED severity. When the EDI-2 was validated in a Spanish population, a mean internal consistency coefficient of 0.63 was found (Garner, 1998).

This article is protected by copyright. All rights reserved. 


\subsubsection{Symptom Check-List 90 Revised (SCL-90-R) (Derogatis, 1990)}

This test consists of 90 items and measures nine primary symptom dimensions and three global indices, being the Global Severity Index, designed to measure overall psychological distress; a Positive Symptom Distress Index, designed to measure the intensity of symptoms; and a Positive Symptom Total (PST), which measures self-reported symptoms. These three measures of global psychopathology were used in our study. The SCL-90 scale has been validated in a Spanish population, obtaining a mean internal consistency coefficient of 0.75 (Derogatis, 2002).

\subsubsection{Difficulties in Emotion Regulation Scale (DERS) (Gratz \& Roemer,} 2004)

The DERS is a 36-item self-report measure that assesses individuals' typical levels of emotion dysregulation across six domains: non-acceptance of emotional responses; difficulties pursuing goal-directed behaviours when experiencing negative emotions; difficulties controlling impulsive behaviours when experiencing negative emotions; lack of emotional awareness; limited access to emotion regulation strategies; and lack of emotional clarity. Higher values indicate greater difficulties in emotion regulation. A Spanish version of the DERS was previously validated in the Spanish population and found to have a satisfactory factorial fit (Gómez-Simón, Penelo, \& de la Osa, 2014; Wolz et al., 2015).

\subsection{Statistical analysis}

Statistical analysis was carried out with Stata15 for Windows. First, the comparison of the prevalence of lifetime NSSI between the groups (HC vs. ED) was based on chi-square tests $\left(\chi^{2}\right)$, and the effect size for the prevalence differences was estimated with the Cohen's- $d$ index $(|d|>0.20$ was considered poor, $|d|>0.5$ was considered moderate and $|d|>0.8$ was considered high; Kelley \& Preacher, 2012). 
Second, the incremental predictive capacity of FA and emotion regulation above and beyond age and group membership (HC vs. ED) on the presence/absence of lifetime NSSI was obtained through binary logistic regression with two steps: the first step included the independent variables age (years-old) and the diagnosis (HC versus $\mathrm{ED}$ ), while the second step added FA (absent versus present) and DERS total score. An additional model included a third block to assess the existence of a potential interaction between DERS and FA on the prediction of the lifetime NSSI, but it was excluded from the final modeling due to the lack of statistical significance of the interaction parameter. The fitting of the regression models was valued with the Hosmer-Lemeshow test (goodness-of-fit was considered for $p>0.05$ ) and the incremental predictive capacity of FA and emotion regulation through the increase of the pseudo-Nagelkerke's $\mathrm{R}^{2}$ coefficient $\left(\Delta \mathrm{R}^{2}\right.$, comparing first and second blocks).

\section{Results}

\subsection{Sample characteristics}

Table 1 includes the frequency distribution of the study variables, stratified by group (HC vs. ED). Table S1 (supplementary material) includes the sociodemographic characteristics of the sample. There were a total of 220 women with ED and 121 female HC in the study. Table S2 (supplementary material) includes a comparison between controls and the eating disorders group

\section{--- Insert Table 1 ---}

\subsection{Associations between food addition with lifetime NSSI and DERS}

In both groups (ED and HC), the prevalence of lifetime NSSI was higher for individuals who met criteria for FA. In the ED sample, the prevalence was $26.4 \%$ (95\%CI: $14.6 \%$ to $38.3 \%$ ) for patients who did not met FA criteria and 40.7\% (95\%CI: $33.3 \%$ to $48.2 \%$ ) for patients

with FA $\left(\chi^{2}=5.69, d f=1, p=.017\right)$. In $\mathrm{HC}$, the prevalence was $17.2 \%$ (95\% CI: $10.4 \%$ to 
$24.1 \%$ ) for patients without FA and $60.0 \%$ (95\%CI: $38.5 \%$ to $81.5 \%$ ) for patients with FA $\left(\chi^{2}=3.89, d f=1, p=.049\right)$. Figure 1 includes the prevalence of lifetime NSSI and the mean values in the DERS-total score stratified by group, as well the results of significance tests and the Cohen's-d estimating the effect sizes for the prevalence differences and the mean differences comparing patients with and without FA. In the BN group, the presence of FA was related to a higher prevalence of lifetime NSSI. In the OSFED-restrictive and OSFEDbinge/purgative subsamples, the presence of FA was linked to increased mean scores on the DERS total score. In the HC group, the presence of FA was related to a higher prevalence of lifetime NSSI and a higher mean score on the DERS total score.

The association between the presence of FA and the DERS-total score was high in the ED group (Pearson's correlation $r=.52, p<.001$ ) as well as in the HC group (Pearson's correlation $r=.327, p<.001)$.

\subsection{Incremental predictive capacity of FA}

Table 2 contains the results of two logistic regressions with two steps, valuing the increase in the predictive capacity due to the presence/absence of FA and emotional dysregulation measures (step 2) after controlling for the patients' age and the diagnostic subtype (step 1). Separate models for FA and DERS measures were obtained due to the high correlation between these variables in the sample. The presence of FA was a significant positive predictor of the presence of lifetime NSSI, with a specific predictive capacity around $3 \%$. Also, the DERS total score was a positive predictor of the presence of NSSI, higher DERStotal scores were related to a higher risk of lifetime NSSI (predictive accuracy 6.4\%). The interaction between the presence of FA and the DERS total score was not significantly predictive for lifetime NSSI $(\mathrm{p}=.666)$.

--- Insert Table 2 --- 


\section{Discussion}

In this study, we investigated the prevalence of NSSI in ED patients and HC with and without FA. Furthermore, we investigated the prevalence of NSSI among different ED subtypes with and without FA. Additionally, we were interested in exploring the interaction between presence of FA, NSSI and emotion dysregulation. Using a predictive model, we could discern whether FA and emotional dysregulation was a predictor of the presence of lifetime NSSI.

As expected, based on previous studies, the prevalence of FA was significantly higher in ED patients as compared to healthy controls. FA prevalence was highest in binge eating/purging-type of ED including bulimia nervosa (BN) and binge eating disorder (BED) (Granero et al., 2014, 2018). Interestingly, there was also a high prevalence of FA in patients with AN-binge/purge subtype.

Also, DERS total scores were significantly higher among ED patients as compared to controls. Both FA and lifetime NSSI prevalence were significantly $(\mathrm{p}<.001)$ higher amongst ED patients as compared to HC. Also in previous studies, emotion dysregulation has been positively associated with the symptom count on the YFAS, which supports the existence of emotional dysregulation in individuals with FA (Pivarunas \& Conner, 2015). Given the overlap of increased FA and NSSI prevalence in the ED group as well as higher DERS scores amongst the ED patients, it could be hypothesized that emotion dysregulation likely serves as a mediator between FA and NSSI, though future studies would be needed to test such interactions.

Previous longitudinal studies investigating NSSI risk factors in the general population have revealed that a prior history of NSSI was the only clinically significant predictor of future NSSI (Fox et al., 2015). Cross-sectional studies investigating risk for NSSI have revealed that individuals with anxiety and mood disorders were at greater risk for 
engagement in NSSI than individuals without a diagnosis (Bentley, Cassiello-Robbins, Vittorio, Sauer-Zavala, \& Barlow, 2015). The predictive model in the study at hand revealed the presence of FA as a predictor of NSSI independent of group assignment, ED diagnosis, and age (Claes, Luyckx, et al., 2015). The high association of FA and NSSI when controlling for confounders reveals a novel relationship that serves as an important launching pad for future studies. As evidenced by prior research revealing endorsed feelings of loss of control amongst individuals with NSSI and FA, this finding further supports the potential existence of a shared underlying etiology (Claes, Islam, et al., 2015; Wolz et al., 2017). Furthermore, emotion dysregulation, as measured by DERS scores was predictive of NSSI, controlling for age and group. There is a need for increasing awareness about the role of lack of effective strategies for dealing with emotions as triggering factors for self-harming behaviors and maladaptive food intakes.

\subsection{Limitations}

There are several limitations to our study. The cross-sectional nature of our study design serves as a hindrance to establishing causality between FA, ED diagnosis, and NSSI. Future studies should investigate the temporal dynamics of FA, emotion regulation and NSSI, to better understand if NSSI behaviors are exhibited in correspondence with the experience of symptoms. Another limitation of the study at hand is the limited sample size, especially amongst comparison groups, and the lack of males in the sample.

The debate over the existence of FA as a substance-use disorder versus a behavioral disorder is ongoing. Individuals in support of the substance abuse framework of FA (and the framework in which the YFAS 2.0 is rooted) posit that specific components of certain foods elicit addictive-like eating (Schulte, Potenza, \& Gearhardt, 2016), whereas others argue that FA is a behavior-based addiction, rather than one rooted in the food substance itself (Hebebrand et al., 2014). With the definition of FA not unanimously agreed upon, the use and 
definition of YFAS 2.0 as a metric of FA in this study serves as a potential limitation. Finally, the one-item diagnostic tool for assessing lifetime NSSI is limited to only providing a dichotomous result of each behavior (Claes, Klonsky, Muehlenkamp, Kuppens, \& Vandereycken, 2010) and the possibility exists that participants may have engaged in NSSI in the past but that this behavior is not currently present. As such, it would be beneficial for future study to expand the scope of NSSI and FA measurement tools used in the study at hand and to explore whether NSSI precedes or proceeds the emergence of FA. There is the likelihood that NSSI could be an episodic behavior which particularly effected by emotions as evidenced by its relation to emotional dysregulation whereas FA symptomology may be more enduring.

\section{Conclusion}

As evidenced by the higher prevalence of NSSI in binge-type eating disorders, paired with FA and DERS scores being significant predictors of NSSI in our model, we conclude the likelihood of a shared underlying etiology between emotional dysregulation, FA, and NSSI. It is possible that this shared etiology is explained, in part, by emotion regulation deficits and shared feelings of loss of control and in binge-type ED, FA, and NSSI populations. Furthermore, our results confirm earlier findings of elevated prevalence of NSSI amongst individuals with binge-type ED (Vieira et al., 2017). Future studies should confirm these findings, as they may be of clinical relevance; these results hold the potential to allow for more targeted treatment for individuals who present NSSI. Specifically, our results highlight the potential importance of treating emotion dysregulation in FA, ED, and NSSI populations in the clinical setting. 


\section{References:}

American Psychiatric Association. (2013). Diagnostic and Statistical Manual of Mental Disorders, Fifth Edition. Washington, DC: American Psychiatric Association.

Andover, M. S., \& Morris, B. W. (2014). Expanding and Clarifying the Role of Emotion Regulation in Nonsuicidal Self-Injury. The Canadian Journal of Psychiatry, 59(11), $569-575$.

American Psychiatric Association. (2000). Diagnostic and Statistical Manual of Mental Disorders, Fourth Edition, Text Revision (DSM-IV-TR). Washington, DC: American Psychiatric Association.

Bentley, K. H., Cassiello-Robbins, C. F., Vittorio, L., Sauer-Zavala, S., \& Barlow, D. H. (2015). The association between nonsuicidal self-injury and the emotional disorders: A meta-analytic review. Clinical Psychology Review, 37, 72-88.

Berner, L. A., Crosby, R. D., Cao, L., Engel, S. G., Lavender, J. M., Mitchell, J. E., \& Wonderlich, S. A. (2017). Temporal associations between affective instability and dysregulated eating behavior in bulimia nervosa. Journal of Psychiatric Research, 92, $183-190$.

Claes, L., Islam, M. A., Fagundo, A. B., Jimenez-Murcia, S., Granero, R., Agüera, Z., ... Fernández-Aranda, F. (2015). The Relationship between Non-Suicidal Self-Injury and the UPPS-P Impulsivity Facets in Eating Disorders and Healthy Controls. PLOS ONE, 10(5), e0126083.

Claes, L., Klonsky, E. D., Muehlenkamp, J., Kuppens, P., \& Vandereycken, W. (2010). The affect-regulation function of nonsuicidal self-injury in eating-disordered patients: which affect states are regulated? Comprehensive Psychiatry, 51(4), 386-392.

Claes, L., Luyckx, K., Bijttebier, P., Turner, B., Ghandi, A., Smets, J., ... Schoevaerts, K. (2015). Non-suicidal self-injury in patients with eating disorder: Associations with 
identity formation above and beyond anxiety and depression. European Eating Disorders Review, 23(2), 147-155.

Claes, L., \& Muehlenkamp, J. J. (2014). Non-suicidal Self-Injury and Eating Disorders: Dimensions of Self-Harm. In Non-Suicidal Self-Injury in Eating Disorders (pp. 3-18). Berlin, Heidelberg: Springer Berlin Heidelberg.

Claes, L., Soenens, B., Vansteenkiste, M., \& Vandereycken, W. (2012). The scars of the inner critic: Perfectionism and nonsuicidal self-injury in eating disorders. European Eating Disorders Review, 20(3), 196-202.

Derogatis, L. (1990). SCL-90-R. Administration, scoring and procedures manual (Clinical P). Baltimore, MD.

Derogatis, L. (2002). SCL-90-R: cuestionario de 90 síntomas: manual. Madrid: TEA Editorial.

First, M. B., Spitzer, R. L., Gibbon, M., \& Williams, J. B. W. (2002). Structured Clinical Interview for DSM-IV-TR Axis I Disorders, Patient Edition (SCID-I/P, 11/2002 revision). for DSMIV.

Fox, K. R., Franklin, J. C., Ribeiro, J. D., Kleiman, E. M., Bentley, K. H., \& Nock, M. K. (2015). Meta-analysis of risk factors for nonsuicidal self-injury. Clinical Psychology Review, 42, 156-167.

Garner, D. (1998). Inventario de Trastornos de la Conducta Alimentaria (EDI-2)-Manual (TEA). Madrid: TEA.

Garner, D. M. (1991). Eating Disorder Inventory-2. Book, Odessa: Psychological Assessment Resources.

Gearhardt, A. N., Corbin, W. R., \& Brownell, K. D. (2016). Development of the Yale Food Addiction Scale Version 2.0. Psychology of Addictive Behaviors, 30(1), 113-121.

Gearhardt, A. N., Davis, C., Kuschner, R., \& Brownell, K. D. (2011). The addiction potential 
of hyperpalatable foods. Current Drug Abuse Reviews, 4(3), 140-145.

Gearhardt, A. N., White, M. A., Masheb, R. M., Morgan, P. T., Crosby, R. D., \& Grilo, C. M.

(2012). An examination of the food addiction construct in obese patients with binge eating disorder. International Journal of Eating Disorders, 45(5), 657-663.

Gómez-Expósito, A., Wolz, I., Fagundo, A. B., Granero, R., Steward, T., Jiménez-Murcia, S., ... Fernández-Aranda, F. (2016). Correlates of Non-suicidal Self-Injury and Suicide Attempts in Bulimic Spectrum Disorders. Frontiers in Psychology, 7, 1244.

Gómez-Simón, I., Penelo, E., \& de la Osa, N. (2014). Factor structure and measurement invariance of the Difficulties Emotion Regulation Scale (DERS) in Spanish adolescents. Psicothema, 26(3), 401-408.

Granero, R., Hilker, I., Agüera, Z., Jiménez-Murcia, S., Sauchelli, S., Islam, M. A., ... Fernández-Aranda, F. (2014). Food Addiction in a Spanish Sample of Eating Disorders: DSM-5 Diagnostic Subtype Differentiation and Validation Data. European Eating Disorders Review, 22(6), 389-396.

Granero, R., Jiménez-Murcia, S., Gearhardt, A. N., Agüera, Z., Aymamí, N., Gómez-Peña, M., ... Fernández-Aranda, F. (2018). Validation of the Spanish Version of the Yale Food Addiction Scale 2.0 (YFAS 2.0) and Clinical Correlates in a Sample of Eating Disorder, Gambling Disorder, and Healthy Control Participants. Frontiers in Psychiatry, 9, 208.

Gratz, K. L., \& Roemer, L. (2004). Multidimensional Assessment of Emotion Regulation and Dysregulation: Development, Factor Structure, and Initial Validation of the Difficulties in Emotion Regulation Scale. Journal of psychopathology and behavioral assessment, 26(1), 41-54.

Hebebrand, J., Albayrak, Ö., Adan, R., Antel, J., Dieguez, C., de Jong, J., ... Dickson, S. L. (2014). "Eating addiction", rather than "food addiction", better captures addictive-like 
eating behavior. Neuroscience and Biobehavioral Reviews, 47, 295-306.

Islam, M. A., Steiger, H., Jimenez-Murcia, S., Israel, M., Granero, R., Agüera, Z., ... Fernández-Aranda, F. (2015). Non-suicidal Self-injury in Different Eating Disorder Types: Relevance of Personality Traits and Gender. European Eating Disorders Review, 23(6), 553-560.

Kelley, K., \& Preacher, K. J. (2012). On effect size. Psychological Methods, 17(2), 137-152.

Kranzler, A., Fehling, K. B., Anestis, M. D., \& Selby, E. A. (2016). Emotional dysregulation, internalizing symptoms, and self-injurious and suicidal behavior: Structural equation modeling analysis. Death Studies, 40(6), 358-366.

Meule, A., \& Gearhardt, A. (2014). Food Addiction in the Light of DSM-5. Nutrients, 6(9), $3653-3671$.

Meule, A., von Rezori, V., \& Blechert, J. (2014). Food Addiction and Bulimia Nervosa. European Eating Disorders Review, 22(5), 331-337.

Muehlenkamp, J. J., Peat, C. M., Claes, L., \& Smits, D. (2012). Self-injury and disordered eating: Expressing emotion dysregulation through the body. Suicide and LifeThreatening Behavior, 42(4), 416-425.

Nunes-Neto, P. R., Köhler, C. A., Schuch, F. B., Solmi, M., Quevedo, J., Maes, M., ... Carvalho, A. F. (2018). Food addiction: Prevalence, psychopathological correlates and associations with quality of life in a large sample. Journal of Psychiatric Research, 96, $145-152$.

Pisetsky, E. M., Haynos, A. F., Lavender, J. M., Crow, S. J., \& Peterson, C. B. (2017). Associations between emotion regulation difficulties, eating disorder symptoms, nonsuicidal self-injury, and suicide attempts in a heterogeneous eating disorder sample. Comprehensive Psychiatry, 73, 143-150.

Pivarunas, B., \& Conner, B. T. (2015). Impulsivity and emotion dysregulation as predictors 
of food addiction. Eating Behaviors, 19, 9-14.

Schulte, E. M., \& Gearhardt, A. N. (2017). Development of the Modified Yale Food Addiction Scale Version 2.0. European Eating Disorders Review, 25(4), 302-308.

Schulte, E. M., Potenza, M. N., \& Gearhardt, A. N. (2016). A commentary on the "eating addiction" versus "food addiction" perspectives on addictive-like food consumption. Appetite, 115:9-15.

Solano, R., Fernández-Aranda, F., Aitken, A., López, C., \& Vallejo, J. (2005). Self-injurious behaviour in people with eating disorders. European Eating Disorders Review, 13(1), 3 10.

Steward, T., Mestre-Bach, G., Vintró-Alcaraz, C., Lozano-Madrid, M., Agüera, Z., Fernández-Formoso, J., ... Fernández-Aranda, F. (2018). Food Addiction and Impaired Executive Functions in Women with Obesity. European Eating Disorders Review. [Epub ahead of print].

Svirko, E., \& Hawton, K. (2007). Self-Injurious Behavior and Eating Disorders: The Extent and Nature of the Association. Suicide and Life-Threatening Behavior, 37(4), 409-421.

Vieira, A. I., Machado, B. C., Machado, P. P. P., Brandão, I., Roma-Torres, A., \& Gonçalves, S. (2017). Putative Risk Factors for Non-Suicidal Self-Injury in Eating Disorders. European Eating Disorders Review, 25(6), 544-550.

Vieira, A. I., Machado, B. C., Moreira, C. S., Machado, P. P. P., Brandão, I., Roma-Torres, A., \& Gonçalves, S. (2018). Eating disorders and non-suicidal self-injury: Structural equation modelling of a conceptual model. European Eating Disorders Review, 26(5), 431-437.

Wolz, I., Agüera, Z., Granero, R., Jiménez-Murcia, S., Gratz, K. L., Menchón, J. M., \& Fernández-Aranda, F. (2015). Emotion regulation in disordered eating: Psychometric properties of the difficulties in emotion regulation scale among spanish adults and its 
interrelations with personality and clinical severity. Frontiers in Psychology, 6:907.

Wolz, I., Granero, R., \& Fernández-Aranda, F. (2017). A comprehensive model of food addiction in patients with binge-eating symptomatology: The essential role of negative urgency. Comprehensive Psychiatry, 74, 118-124.

Wolz, I., Hilker, I., Granero, R., Jiménez-Murcia, S., Gearhardt, A. N., Dieguez, C., ... Fernández-Aranda, F. (2016). "Food Addiction" in Patients with Eating Disorders is Associated with Negative Urgency and Difficulties to Focus on Long-Term Goals. Frontiers in Psychology, 7, 61.

Yurkowski, K., Martin, J., Levesque, C., Bureau, J.-F., Lafontaine, M.-F., \& Cloutier, P. (2017). Emotion dysregulation mediates the influence of relationship difficulties on nonsuicidal self-injury behavior in young adults. Psychiatry research, 228(3), 871-878.

Zetterqvist, M. (2015). The DSM-5 diagnosis of nonsuicidal self-injury disorder: a review of the empirical literature. Child and Adolescent Psychiatry and Mental Health, 9, 31. 
Table 1 Sample description stratified by group

\begin{tabular}{|c|c|c|c|c|c|c|c|c|c|c|c|c|c|c|}
\hline & \multicolumn{2}{|c|}{$\begin{array}{l}\text { Healthy } \\
\text { control } \\
n=121\end{array}$} & \multicolumn{2}{|c|}{$\begin{array}{c}\text { Binge Eating } \\
\text { Disorder } \\
n=36\end{array}$} & \multicolumn{2}{|c|}{$\begin{array}{c}\text { Bulimia } \\
\text { Nervosa } \\
n=59\end{array}$} & \multicolumn{2}{|c|}{$\begin{array}{c}\text { AN } \\
\text { Restrictive } \\
n=24\end{array}$} & \multicolumn{2}{|c|}{$\begin{array}{c}\text { AN Binge- } \\
\text { Eating/Purging } \\
n=19\end{array}$} & \multicolumn{2}{|c|}{$\begin{array}{c}\text { OSFED- } \\
\text { restrictive } \\
n=33\end{array}$} & \multicolumn{2}{|c|}{$\begin{array}{c}\text { OSFED- } \\
\text { binging/purging } \\
n=49\end{array}$} \\
\hline & Mean & $S D$ & Mean & $S D$ & Mean & $S D$ & Mean & $S D$ & Mean & $S D$ & Mean & $S D$ & Mean & $S D$ \\
\hline Age (years-old) & 21.07 & 2.91 & 36.44 & 12.13 & 29.76 & 11.20 & 21.00 & 3.15 & 26.32 & 9.36 & 24.64 & 10.07 & 28.53 & 11.68 \\
\hline $\mathrm{BMI}\left(\mathrm{kg} / \mathrm{m}^{2}\right)$ & 21.76 & 4.16 & 40.49 & 11.05 & 25.87 & 5.24 & 16.16 & 1.42 & 17.07 & 1.05 & 19.21 & 0.91 & 26.21 & 7.73 \\
\hline EDI-2 total score & 33.77 & 25.82 & 112.08 & 31.39 & 117.95 & 37.16 & 82.42 & 44.25 & 100.63 & 36.15 & 86.66 & 48.71 & 106.45 & 41.28 \\
\hline SCL-90R GSI & 0.64 & 0.46 & 1.71 & 0.71 & 1.85 & 0.61 & 1.64 & 0.72 & 1.73 & 0.64 & 1.58 & 0.86 & 1.76 & 0.85 \\
\hline YFAS-2 total criteria & 0.93 & 1.98 & 8.86 & 2.88 & 9.31 & 2.14 & 3.17 & 2.28 & 7.79 & 3.77 & 4.36 & 3.54 & 5.51 & 3.54 \\
\hline Emotion regulation & Mean & $S D$ & Mean & $S D$ & Mean & $S D$ & Mean & $S D$ & Mean & $S D$ & Mean & $S D$ & Mean & $S D$ \\
\hline DERS Non-acceptance & 12.74 & 5.62 & 19.26 & 6.29 & 20.38 & 6.95 & 18.82 & 6.47 & 19.25 & 5.87 & 18.52 & 6.61 & 18.02 & 8.13 \\
\hline DERS Goal directed behavior & 12.61 & 4.20 & 18.00 & 4.83 & 18.40 & 5.25 & 16.77 & 4.50 & 17.88 & 4.76 & 15.84 & 6.03 & 17.33 & 5.57 \\
\hline DERS Impulse control & 10.81 & 4.26 & 16.13 & 6.25 & 18.85 & 6.50 & 15.14 & 6.36 & 16.50 & 6.76 & 14.29 & 5.92 & 16.52 & 6.53 \\
\hline DERS Emotional awareness & 14.73 & 4.00 & 16.39 & 5.36 & 18.09 & 4.60 & 18.95 & 4.72 & 17.69 & 5.72 & 17.81 & 4.45 & 16.83 & 5.46 \\
\hline DERS Regulation strategies & 15.01 & 5.61 & 24.13 & 7.70 & 27.53 & 8.18 & 25.64 & 8.44 & 26.88 & 8.10 & 21.94 & 8.71 & 24.90 & 8.45 \\
\hline DERS Emotional clarity & 10.35 & 3.67 & 13.13 & 4.37 & 14.66 & 4.39 & 15.41 & 5.65 & 14.31 & 4.38 & 14.03 & 5.64 & 13.88 & 4.98 \\
\hline DERS Total score & 76.24 & 20.49 & 107.03 & 24.87 & 117.91 & 25.44 & 110.73 & 27.09 & 112.50 & 23.57 & 102.42 & 29.54 & 107.50 & 28.51 \\
\hline Prevalence & $n$ & $\%$ & $n$ & $\%$ & $n$ & $\%$ & $n$ & $\%$ & $n$ & $\%$ & $n$ & $\%$ & $n$ & $\%$ \\
\hline Food addiction & 5 & $4.13 \%$ & 29 & $80.56 \%$ & 56 & $94.92 \%$ & 14 & $58.33 \%$ & 16 & $84.21 \%$ & 19 & $57.58 \%$ & 33 & $67.35 \%$ \\
\hline Lifetime NSSI & 23 & $19.01 \%$ & 10 & $27.78 \%$ & 26 & $44.07 \%$ & 6 & $25.00 \%$ & 7 & $36.84 \%$ & 8 & $24.24 \%$ & 25 & $51.02 \%$ \\
\hline
\end{tabular}

Note. AN: anorexia nervosa. OSFED: other specified feeding eating disorder. SD: standard deviation.

This article is protected by copyright. All rights reserved. 
Table 2 Incremental predictive capacity of food addiction and emotion regulation on the presence of lifetime NSSI: binary logistic regression adjusted for age and diagnosis

\begin{tabular}{|c|c|c|c|c|c|c|c|c|c|}
\hline & & $B$ & SE & $p$ & OR & \multicolumn{2}{|c|}{$95 \% \mathrm{Cl}(\mathrm{OR})$} & $\Delta R^{2}$ & $\mathrm{H}-\mathrm{L}$ \\
\hline \multicolumn{10}{|c|}{ Model 1: Food addiction } \\
\hline \multirow[t]{2}{*}{ First step } & Age (years-old) & -0.013 & 0.013 & .339 & 0.987 & 0.962 & 1.013 & .068 & .086 \\
\hline & Diagnosis $(0=\mathrm{HC} ; 1=\mathrm{ED})$ & 1.121 & 0.298 & $.001^{*}$ & 3.068 & 1.710 & 5.503 & & \\
\hline \multirow[t]{3}{*}{ Second step } & Age (years-old) & -0.019 & 0.014 & .171 & 0.981 & 0.955 & 1.008 & .028 & .151 \\
\hline & Diagnosis $(0=\mathrm{HC} ; 1=\mathrm{ED})$ & 0.552 & 0.382 & .148 & 1.737 & 0.822 & 3.673 & & \\
\hline & FA (0=no; $1=y e s)$ & 0.864 & 0.350 & $.013^{*}$ & 2.374 & 1.196 & 4.710 & & \\
\hline \multicolumn{10}{|l|}{ Model 2: DERS } \\
\hline \multirow[t]{2}{*}{ First step } & Age (years-old) & -0.013 & 0.013 & .339 & 0.987 & 0.962 & 1.013 & .068 & .086 \\
\hline & Diagnosis $(0=\mathrm{HC} ; 1=\mathrm{ED})$ & 1.121 & 0.298 & $.001^{*}$ & 3.068 & 1.710 & 5.503 & & \\
\hline \multirow[t]{3}{*}{ Second step } & Age (years-old) & -0.010 & 0.014 & .470 & 0.990 & 0.964 & 1.017 & & \\
\hline & Diagnosis $(0=\mathrm{HC} ; 1=\mathrm{ED})$ & 0.433 & 0.353 & .220 & 1.542 & 0.772 & 3.081 & .064 & .537 \\
\hline & DERS Total score & 0.020 & 0.005 & $.001^{*}$ & 1.020 & 1.010 & 1.031 & & \\
\hline
\end{tabular}

Note. HC: healthy control. ED: eating disorder. FA: food addiction. Total sample $(n=341)$.

$\Delta R^{2}$ : Change- increase for the second block/step. OR: odds ratio. H-L: Hosmer-Lemeshow test. *Bold: significant parameter (.05 level). 
Figure 1 Prevalence of lifetime NSSI and DERS total scores for patients with and without food addiction: analysis stratified by group. OSFED: other specified feeding eating disorder. 
